\title{
A dinamometria manual e seu uso na avaliação nutricional
}

\author{
Hand grip strength test and its use in \\ nutritional assessment
}

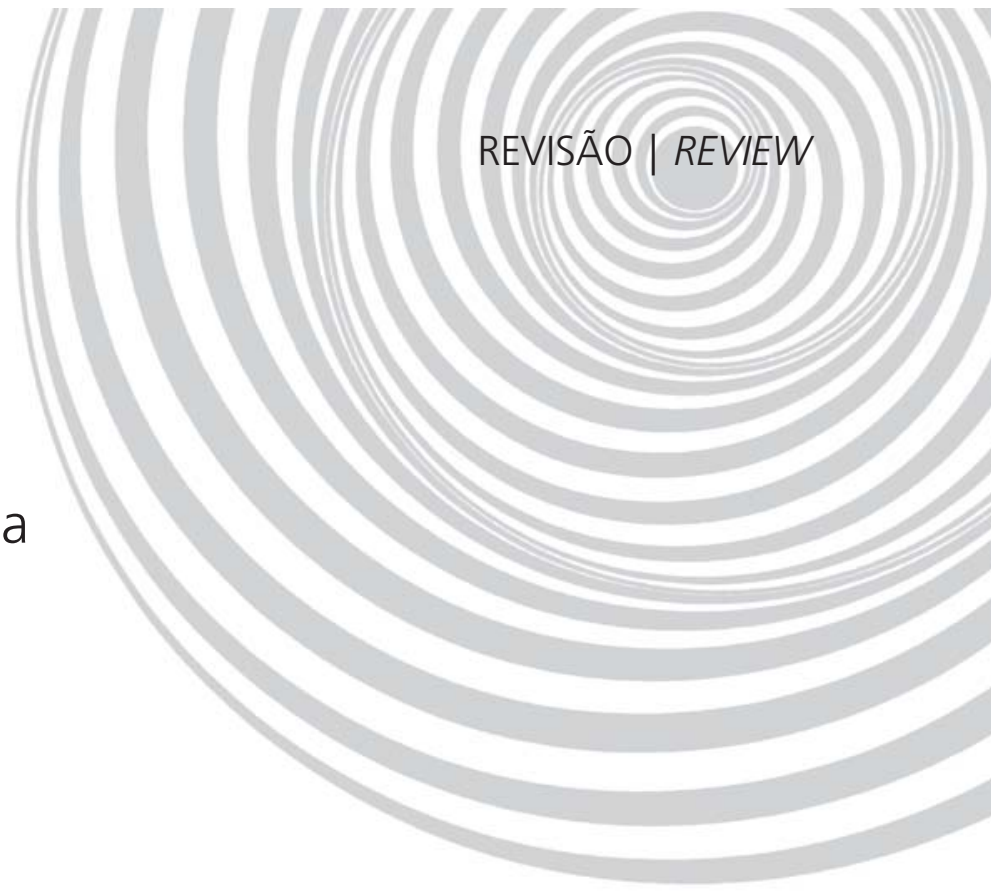

Michael Maia SCHLÜSSEL'

Luiz Antonio dos ANJOS²

Gilberto KAC1

RE S U M O

Esta revisão de literatura aborda aspectos metodológicos e fisiológicos da dinamometria manual. A dinamometria manual é um teste funcional do músculo esquelético que vem recebendo uma crescente atenção de clínicos e pesquisadores da área de saúde nos últimos anos. Recentemente, tem merecido atenção como indicador do estado nutricional, particularmente para pacientes internados. Dentre os principais fatores que influenciam esta medida, destacam-se o sexo, a idade, a estatura, a massa corporal e a mão dominante dos indivíduos. Os resultados desta revisão demonstram ainda que diversos outros fatores, relacionados ao protocolo de aferição, como a posição do indivíduo, o tipo de instrumento utilizado, o número de aferições realizadas, o intervalo de descanso entre as aferições, a presença de estímulo verbal e de um pré-teste, também podem influenciar os valores alcançados por um indivíduo em uma avaliação da dinamometria manual. Dessa forma, é importante que um protocolo de aferição padronizado seja desenvolvido, para que se obtenham medidas válidas de dinamometria manual. Ainda são escassos os estudos que propõem valores de referência para a dinamometria manual e a literatura ainda se ressente de valores de referência baseados em dados obtidos a partir de amostras de base populacional.

Palavras-chave: Dinamotria manual. Estado nutricional. Valores de referência.

A B S T R A C T

This paper reviews the methodological and physiological aspects of hand grip strength measurement. Hand grip strength assesses skeletal muscle function and has received increasing attention from physicians and health-related researchers in the last years. Recently, it has deserved particular attention as an indicator of

\footnotetext{
1 Universidade Federal do Rio de Janeiro, Instituto de Nutrição Josué de Castro, Departamento de Nutrição Social e Aplicada, Programa de Pós-Graduação em Nutrição. Av. Brig. Trompowsky, s/n., Bloco J, $2^{\circ}$ andar, Ilha do Fundão, 21941-590, Rio de Janeiro, RJ, Brasil. Correspondência para/Correspondence to: G. KAC. E-mails: <kacetal@gmail.com>; <gkac@nutricao.ufrj.br>. 2 Universidade Federal Fluminense, Departamento de Nutrição Social, Laboratório de Avaliação Nutricional e Funcional. Niterói, RJ, Brasil.
} 
224 | M.M. SCHLÜSSEL et al.

nutritional status, particularly in hospitalized patients. Sex, age, height, body mass and the dominant hand of the subjects are the main factors that influence the measure. The results of this review demonstrate that many other factors associated with the assessment protocol, such as the position of the individual, the type of instrument used, the number of measurements performed, the rest periods between measurements, the presence of verbal stimulation and a warm-up prior to the test can also influence the results. Therefore, it is important to develop a standardized protocol to improve the reliability of the measurements for this variable. Normative data for hand grip strength are still scarce and the literature still resents from the existence of reference values from population-based studies.

Indexing terms: Handgrip strength. Nutritional status. Reference values.

\section{N T R O D U ÇÃ O}

A aferição da força máxima voluntária de preensão manual, ou simplesmente dinamometria manual (DM), consiste em um teste simples e objetivo que tem como princípio estimar a função do músculo esquelético'. A consistência interna das medidas de força exercidas por diferentes grupamentos musculares sustenta a utilização da DM para caracterizar o status funcional muscular geral ${ }^{2}$. Trata-se de um teste realizado geralmente com um aparelho portátil - dinamômetro - sendo um procedimento rápido, de baixo custo e pouco invasivo.

O termo força muscular é utilizado para designar a habilidade de um determinado músculo, ou grupamento muscular, em produzir ou resistir a uma força; podendo ser classificada como isométrica, isocinética ou isotônica. A DM é uma medida de força isométrica, que envolve o emprego de força sobre um objeto imóvel. O músculo se contrai, permanecendo sob tensão constante por um curto intervalo de tempo, porém, há pouca alteração em seu comprimento.

A aplicação clínica da DM tem merecido maior atenção nos últimos anos ${ }^{3}$ e é feita em diversos campos da área da saúde. Amplamente utilizada na área de reabilitação por terapeutas ocupacionais, fisioterapeutas e médicos; a DM vem sendo reconhecida como um instrumento útil de avaliação funcional, seja no acompanhamento do estado nutricional de pacientes cirúrgicos ${ }^{4}$ ou na avaliação funcional da população idosa ${ }^{2,5}$.

Este trabalho apresenta e discute as informações metodológicas e fisiológicas a respeito deste teste de avaliação funcional. Além disso, fez-se uma revisão sistemática sobre o uso da DM na avaliação nutricional. O artigo está organizado de forma a incluir informações a respeito dos diferentes instrumentos utilizados: protocolo de aferição (instruções, posição do indivíduo, posição de manuseio, número de aferições, duração da contração, períodos de descanso, pré-teste), valores de referência disponíveis e características individuais que influenciam os valores de DM (sexo, idade, estatura, massa corporal, mão dominante), parâmetros importantes a serem considerados caso a DM seja incorporada aos protocolos de rotina para avaliação do estado nutricional e funcional.

\section{Tipos de dinamômetros}

Inúmeros instrumentos estão disponíveis para aferir valores de DM. Os aparelhos utilizados para esta medida de força podem ser classificados em quatro categorias: hidráulicos, pneumáticos, mecânicos e strain gauges (ou células de carga).

Dinamômetros hidráulicos são sistemas selados, que medem a DM em quilogramas (ou libras) força. Instrumentos pneumáticos usam um mecanismo de compressão em uma bolsa de ar para determinar a DM; são normalmente utilizados em indivíduos que apresentam dor e favorecer a medida em milímetros de mercúrio ou libra/polegada². Dinamômetros mecânicos são instrumentos que medem a DM em função da quantidade de tensão produzida em uma mola de aço. Já os strain gauges são aparelhos em que a força empreendida em uma célula de carga é captada eletronicamente, amplificada e transmitida para um monitor digital ${ }^{6}$. 
Diversos modelos de dinamômetro mecânico têm sido utilizados e, aparentemente, este é o tipo de dinamômetro portátil mais confortável e de fácil manuseio para os indivíduos avaliados. Entre os estudos revisados que utilizaram instrumentos mecânicos, os modelos mais comuns foram os dinamômetros Harpenden ${ }^{7-9}$ e Smedley ${ }^{10-12}$. Entretanto, o modelo hidráulico do dinamômetro Jamar aparece como sendo o instrumento mais amplamente utilizado em estudos que medem valores de $\mathrm{DM}^{13-20}$.

Desenvolvido por Bechtol21 , em 1954, o dinamômetro Jamar é um aparelho de simples manuseio, fornecendo uma leitura rápida e direta, além de permitir fácil utilização tanto em estudos de campo quanto em situações clínicas ambulatoriais. O modelo hidráulico do dinamômetro Jamar é o recomendado pela Sociedade Americana de Terapeutas da Mão (American Society of Hand Therapists - ASHT), sendo considerado o mais acurado e preciso instrumento para avaliar a $\mathrm{DM}^{13}$

A acurácia do aparelho deve ser mantida por meio de calibração regular. É recomendado que a calibração do dinamômetro Jamar seja checada ao menos uma vez por ano e, mais freqüentemente (entre 4 e 6 meses), quando utilizado diariamente. Contudo, parece não haver estudos examinando por quanto tempo o dinamômetro Jamar, ou qualquer outro, mantém sua acurácia, indicando, desta forma, o tempo ótimo de recalibraçãó.

\section{Posição do indivíduo durante a aferição}

É consenso que, ao realizar a aferição de parâmetros biológicos em um indivíduo, um protocolo de teste deve ser padronizado para assegurar a confiabilidade e comparabilidade das medidas. Um protocolo de teste compreende as instruções fornecidas e os procedimentos adotados para a coleta dos dados. No caso particular da DM, entre estes procedimentos inclui-se a posição do indivíduo durante a aferição. Entretanto, diferentes posições e protocolos têm sido observados para a aferição da DM.
Alguns dos estudos revisados usam a posição padronizada proposta pela ASHT 14,15,17,22 . Esta posição é descrita por Innes $^{6}$ como:

[...] sentado em uma cadeira com encosto reto e sem suporte para os braços, ombro aduzido e neutralmente rodado, cotovelo flexionado a $90^{\circ}$, antebraço em posição neutra e punho entre $0^{\circ}$ e $30^{\circ}$ de extensão e $0^{\circ}$ e $15^{\circ}$ de desvio ulnar.

Esta posição foi desenvolvida para o uso do modelo hidráulico do dinamômetro Jamar. Contudo, há estudos nos quais pequenas variações nessa posição foram observadas, como, por exemplo: permitiu-se que a posição do punho fosse auto-selecionada pelos indivíduos, para que obtivessem a força máxima de preensão ${ }^{16}$; ou ainda que alguns indivíduos se posicionassem de pé, ao invés de sentados, mas mantendo a posição padrão para o braço ${ }^{18}$. Outros estudos simplesmente não relatam a posição utilizada, ou o fazem de forma incompleta $7,13,21,23$.

Segundo Innes ${ }^{6}$, variações em relação à posição proposta pela ASHT afetam a medida de DM de algumas maneiras: (i) Posicionar-se de pé, ao invés de sentado, resulta em maiores valores de $\mathrm{DM}$, usando o mesmo instrumento; (ii) $\mathrm{O}$ ombro flexionado a $180^{\circ}$ possibilita o melhor resultado, quando comparado à flexão de $90^{\circ}$ ou a posição padrão $0^{\circ}$; (iii) Estudos sobre o efeito da posição do cotovelo durante a aferição da DM têm apresentado resultados conflitantes. Alguns demonstram a maior medida quando o cotovelo está completamente estendido $\left(0^{\circ}\right)$, outros quando há uma flexão de $90^{\circ}$ e outros, ainda, não encontraram relação entre a posição do cotovelo e a medida de DM.

Hillman et al. ${ }^{24}$, em estudo desenvolvido para propor uma postura prática para aplicação clínica, observaram os maiores valores de DM entre indivíduos saudáveis que se posicionaram sentados em uma cadeira sem suporte para os braços. Entretanto, as comparações foram feitas apenas com as medidas obtidas em indivíduos sentados com os braços apoiados ou recostados a $30^{\circ}$ em uma cama. 
Logo, percebe-se que variações na posição usando o mesmo instrumento podem influenciar significativamente os resultados e, desta forma, deve-se manter a consistência e a padronização dos procedimentos de aferição. Além disso, é importante atentar para o fato de que a posição durante a aferição pode não depender apenas da vontade do indivíduo ou de um protocolo de teste. Principalmente para a aplicação clínica, a viabilidade de estabelecer a melhor postura para realizar a aferição pode variar, dependendo da morbidade e de seu estágio. Já para a população saudável, particularmente em inquéritos domiciliares, a postura mais prática parece ser a de pé com os braços estendidos ao longo do corpo. De qualquer forma, isso implica em uma dificuldade de estabelecer uma posição padrão, bem como, exige um cuidado do profissional de saúde/pesquisadores ao comparar as medidas observadas com valores de referência.

\section{Posição de manuseio}

Alguns instrumentos, como o dinamômetro Jamar, permitem que se ajuste a pega ao realizar o teste de DM. Isto quer dizer que é possível ajustar a empunhadura (ou posição de manuseio) do dinamômetro, para que melhor se adapte ao tamanho da mão e ao comprimento dos dedos de um indivíduo.

Primeiramente discutidas por Bechtol ${ }^{21}$, as posições de manuseio mais utilizadas nos estudos revisados, particularmente nos estudos que se propõem a fornecer valores normativos para esta medida, são a segunda e a terceira posição do dinamômetro Jamar ${ }^{13,14,16}$, que possui ajuste para cinco posições diferentes.

Segundo Innes ${ }^{6}$, a ASHT recomenda que o dinamômetro Jamar seja ajustado na segunda posição para aferição da DM. Entretanto, a maioria dos estudos não faz menção em relação à posição do dinamômetro utilizada durante a aferição desta medida.

Boadella et al. ${ }^{20}$ ressaltaram a capacidade dos indivíduos em auto-selecionar a posição que
Ihes fornecia o melhor desempenho para realizar o teste de DM. Tanto para os indivíduos que optaram por realizar sentados, quanto para os que realizaram de pé, as maiores leituras de DM observadas foram obtidas na posição auto-selecionada. Os maiores valores absolutos foram observados para as medidas realizadas com os indivíduos de pé.

\section{Instruções}

Tão importante quanto a posição é a determinação de uma seqüência clara de instruções que serão dadas aos indivíduos a serem avaliados. Deve-se procurar optar sempre pelo menor número de instruções e, da mesma forma, que estas sejam passadas da maneira mais simples e objetiva possível.

Também é importante o tom de voz com que essas instruções são transmitidas. Johansson et al. ${ }^{25}$ encontraram uma relação significante entre o volume de voz com o qual as instruções eram transmitidas aos indivíduos e a força de contração isométrica exercida, de modo que volumes aumentados resultavam em valores de força mais elevados durante a contração. Essa diferença pode estar relacionada à motivação com que o indivíduo realiza a medida de força, já que o procedimento é considerado como uma medida de desempenho e que pode ser melhorada. A maioria dos estudos não especifica as instruções dadas aos indivíduos durante a realização dos procedimentos para a coleta de dados de DM. Entre os poucos que o fazem, alguns ressaltam o fato de que "nenhum encorajamento verbal" foi feito ${ }^{19,20}$, enquanto que outros deixam claro que isso foi feito ${ }^{13,15,18}$.

Independentemente de incluir ou não o estímulo verbal durante o procedimento de medição, principalmente para pacientes internados, é de extrema importância que o indivíduo avaliado esteja consciente de que uma medida de força depende, basicamente, de sua disposição em cooperar e reproduzir com eficiência o seu esforço máximo. Da mesma forma, é importante que o 
avaliador esteja consciente de que, intencionalmente ou não, o tom e o volume da voz com que as instruções são transmitidas podem influenciar os resultados. Dessa forma, deve-se procurar usar sempre a mesma intensidade ao instruir o indivíduo que se pretende avaliar.

\section{Número de aferições, duração da contra- ção, períodos de descanso e pré-teste}

Além do cálculo da média de várias leituras de DM, geralmente três, pode-se ainda usar a medida: (i) apenas de uma leitura; (ii) maior entre duas ou três leituras; (iii) média das duas maiores entre três leituras. Segundo Innes ${ }^{6}$, embora todas essas alternativas apresentem boa confiabilidade (coeficiente de correlação intraclasse $>0,93$ ), não foram observadas diferenças significantes entre elas, dando margem para que o avaliador escolha o método que lhe pareça mais adequado. Aparentemente, o procedimento mais comum observado nos estudos revisados é o registro da maior entre três leituras $7,17,18,22,23$.

Sugere-se que um período de contração muscular contínua de 3 segundos seja o suficiente para registrar a máxima leitura de $\mathrm{DM}^{6}$. Além disso, é importante atentar para o fato de que, quando o objetivo é avaliar medidas repetidas de esforço, um período de descanso entre as aferições deve ser respeitado para evitar o efeito da fadiga muscular. A maioria dos estudos revisados utiliza um período de descanso entre as medidas de, no mínimo, 1 minuto 7,17,19,24-26. Porém, intervalos menores também foram observados: 2 a 5 segundos $^{20,23} ; 15$ segundos $^{18}$.

Em estudo realizado por Trossman \& $\mathrm{Li}^{27}$, nenhuma diferença significante foi observada entre os períodos de descanso de 15, 30 e 60 segundos; embora tenha sido notada uma tendência de declínio nas medidas de força ao longo das aferições. $O$ período de 60 segundos, entretanto, teve a menor percentagem de declínio da primeira para a última medida e o maior coeficiente de correlação intraclasse. Dessa forma, os autores recomendam um período de descanso entre as aferições de, no mínimo, um minuto.
Pré-testes na forma de medidas sub-máxima têm sido apontados como uma prática que, quando incorporada ao protocolo, resulta em melhor desempenho durante a aferição da $\mathrm{DM}^{6}$. Isso, possivelmente, está relacionado ao fato de que o indivíduo a ser avaliado pode não estar familiarizado com o aparelho. Dessa forma, se obteria melhor desempenho tendo a oportunidade de testar o dinamômetro e ajustar a melhor empunhadura, antes de realizar a medida de DM máxima propriamente dita.

\section{Influência da dominância}

Como a geração de força depende da área transversal do músculo e como esta depende do treinamento a que o músculo é submetido, é fácil imaginar que possam haver diferenças na DM entre os lados do corpo, dependendo da mão de preferência do indivíduo para realizar tarefas cotidianas, como comer, escrever e carregar pesos. Uma diferença de 10\% na força entre a mão dominante e a não dominante foi descrita nos anos 50 , derivada de uma pesquisa realizada no exército canadense, durante a Primeira Guerra Mundial ${ }^{13}$. Entretanto, estudos que se propuseram a quantificar a diferença de força por dominância, não confirmaram esta "regra de 10\%".

Bechtol ${ }^{21}$, em 1954, relatou uma diferença de força entre a mão dominante e a não dominante que variava de $5,0 \%$ a $10,0 \%$ para a maioria dos indivíduos, podendo chegar a 30,0\% em alguns indivíduos. Schmidt \& Toews ${ }^{13}$, em 1970, apresentaram os resultados de DM obtidos de uma amostra composta por 1128 homens e 80 mulheres, candidatos a vagas como empregados de uma fábrica de aço, e observaram que 97,0\% dos homens estudados apresentaram diferença nos valores de força entre a mão dominante e a não dominante menor do que 10,0\% (em média 3,2\%). Além disso, 22,6\% dos homens apresentaram maiores valores de DM para a mão não dominante e 5,4\%, não apresentavam diferença significante entre as mãos. Entre as mulheres, 20,0\% apresentaram maiores valores de DM para a mão não dominante. 
Outros estudos demonstraram diferença de DM significante entre a mão dominante e a não dominante, mas somente para destros. Crosby et al. ${ }^{16}$ observaram, para a maioria dos indivíduos destros, diferença de $10,0 \%$ a mais na força da mão dominante em relação à não dominante. Entretanto, indivíduos canhotos, aparentemente, não apresentaram diferença de força entre as mãos. Na verdade, 50,0\% dos canhotos e 9,0\% dos destros apresentaram a mão dominante mais fraca, embora essa diferença não fosse estatisticamente significante. Armstrong \& Oldham ${ }^{22}$ observaram diferença estatisticamente significante, mas clinicamente insignificante, de apenas $0,1 \%$ entre as medidas de força da mão dominante e não dominante para indivíduos destros e não observaram diferença significante entre os indivíduos canhotos. Incel et al. ${ }^{19}$ observaram também diferença significativa para DM de indivíduos destros (8,2\%), mas não para canhotos $(3,2 \%)$.

A justificativa para essas observações seria dada, em parte, pelo fato de que indivíduos canhotos vivem em um mundo desenvolvido para pessoas destras. Dessa forma, seriam obrigados a utilizar a mão direita para realizar diversas tarefas cotidianas que, naturalmente, seriam feitas com a mão dominante ${ }^{16}$. Além disso, indivíduos canhotos possuem uma organização funcional dos dois hemisférios do cérebro mais simétrica, em contraste à lateralização cerebral (organização funcional desigual) comumente reconhecida em indivíduos destros ${ }^{18}$. Assim, isso poderia contribuir para um maior desenvolvimento da força na mão não dominante, diminuindo a diferença de força entre as mãos, em indivíduos canhotos.

Vale ressaltar ainda que, em estudo realizado por Hanten et al. ${ }^{18}$, os valores de DM observados para a mão direita foram significantemente maiores do que aqueles observados para a mão esquerda, independentemente da dominância. Esses resultados são concordantes com os resultados de outro estudo, realizado por Mathiowetz et al. ${ }^{14}$, no qual, primeiramente, se questionou a relevância em apresentar os resultados de DM por dominância. Neste estudo, 6 entre 8 medidas realizadas para avaliar a DM de indivíduos adultos foram maiores para a mão direita, mesmo entre canhotos. Dessa forma, estes autores propõem que os valores de DM sejam apresentados não em termos de mão dominante e não dominante, mas simplesmente como DM direita e esquerda.

\section{Outros fatores que influenciam os valores de DM}

Há algumas outras características individuais que influenciam a medida de DM. Entre elas incluem-se a idade, o sexo, a massa corporal e a estatura. Segundo Innes ${ }^{6}$ tem-se observado que: (i) homens apresentam maiores valores de DM do que as mulheres, independentemente do instrumento utilizado; (ii) a DM tem relação curvilínea com a idade. Observa-se aumento na força conforme a idade aumenta, atingindo um pico entre os 30-45 anos. Subseqüentemente, observa-se declínio nesses valores para indivíduos com idade mais avançada; (iii) existe correlação positiva entre a DM, massa corporal e estatura, em indivíduos saudáveis até $98 \mathrm{~kg}$ de massa corporal e $190 \mathrm{~cm}$ de estatura. O autor não faz comentários a respeito dessa correlação a partir desses valores $^{13}$

A Figura 1, elaborada a partir de dados publicados por Luna-Heredia et al. ${ }^{23}$, exemplifica alguns desses pontos em uma amostra de 517 indivíduos espanhóis com idade entre 17 e 97 anos. É interessante notar que a redução de DM é mais acentuada nos homens $(23,7 \mathrm{~kg})$ do que nas mulheres $(13 \mathrm{~kg})$. Além disso, a diferença de DM entre a mão dominante e a não dominante foi maior do que $10 \%$ até a faixa etária compreendida entre os 50-59 anos, para os homens; e em quase todas as faixas etárias para as mulheres. Verifica-se que essa diferença também diminui com a idade, tanto em homens quanto em mulheres.

O tipo de ocupação e as atividades de lazer praticadas por um indivíduo também são apontados como fatores que influenciam a $\mathrm{DM}^{16,21}$. Segundo Innes ${ }^{6}$, estudos que especificamente examinaram o efeito do tipo de trabalho exercido pelos indivíduos na sua medida de DM têm 


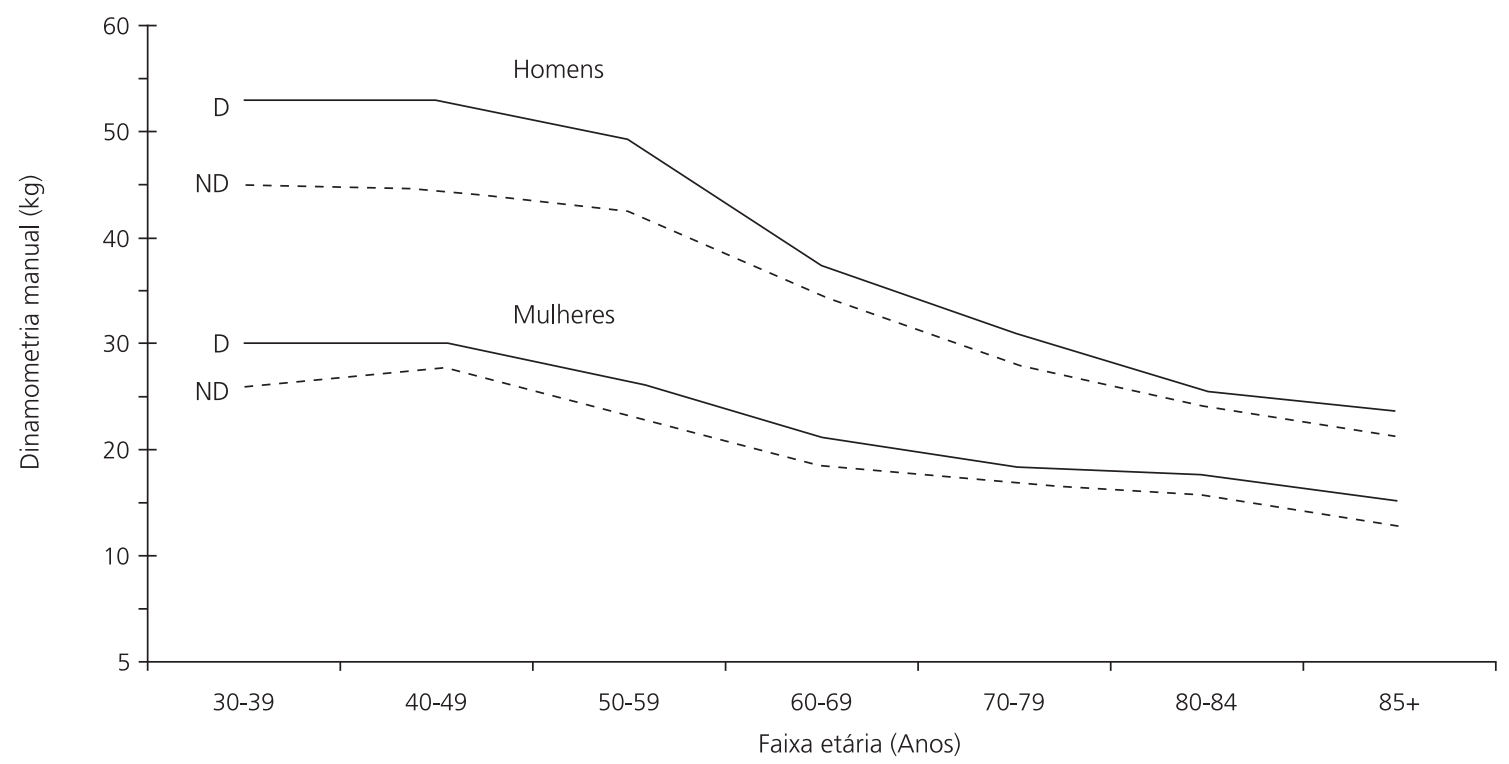

Figura 1. Valores médios de dinamometria manual $(\mathrm{kg})$ em amostra de população espanhola na mão dominante (D) e não dominante (ND). Desenhado a partir de dados publicados por Luna-Heredia et al. ${ }^{23}$.

Nota: D: Mão dominante; ND: Mão não-dominante.

observado resultados conflitantes. Alguns não observaram diferença na DM entre empregados de diferentes áreas de ocupação, enquanto outros observaram que indivíduos que realizavam trabalhos braçais pesados apresentaram maiores valores de DM, seguidos pelos que realizavam trabalhos braçais leves e empregados de escritório.

Atividades de lazer também foram sugeridas como um fator de influência na $\mathrm{DM}^{6}$ e até mesmo, mais importante do que a ocupação do indivíduo ${ }^{16}$. Contudo, essa premissa ainda não foi examinada a fundo, permanecendo apenas a sugestão de que estudos nesse sentido deveriam ser realizados para avaliar o real impacto dessas atividades na DM.

\section{Valores de referência}

A proposta de desenvolver valores de referência consiste em descrever valores típicos ou padrões normativos para uma dada característica, de uma determinada população considerada saudável. Para comparar os dados observados de um indivíduo com valores de referência existentes para uma determinada medida, é impor- tante que sejam utilizados o mesmo instrumento de aferição e o mesmo protocolo de teste. Fatores a serem considerados ao comparar os resultados de uma medida com valores de referência são: a adequação do grupo de referência (por exemplo: sexo e idade), o número de indivíduos que compõem o grupo e quando e onde esses dados foram coletados.

O Quadro 1 ilustra as variações que ocorrem na DM entre indivíduos de diferentes nacionalidades, classificados em dois grandes grupos etários. Uma análise dos valores de referência indica que existe grande variação dos dados, de acordo com a nacionalidade dos indivíduos avaliados nos estudos. Essas diferenças podem ser devidas a diversos fatores como: (i) a etnia das populações de onde as amostras são extraídas (por exemplo: a maior diferença é observada entre os estudos da Austrália ${ }^{28}$ e Nova Zelândia ${ }^{29}$, países próximos geograficamente, mas que possuem populações de origem étnica distinta); (ii) possíveis diferenças nos modelos do dinamômetro utilizado; (iii) calibrações inadequadas dos instrumentos ${ }^{6}$; (iv) características da amostra (por exemplo: padrão 
Quadro 1. Variações nos valores de referência para dinamometria manual ( $\mathrm{kg}$ ) para indivíduos (homens e mulheres) de 20 e 45 anos de diferentes nacionalidades.

\begin{tabular}{|c|c|c|c|c|}
\hline \multirow{2}{*}{ País (referência) } & \multicolumn{2}{|c|}{ Homens } & \multicolumn{2}{|c|}{ Mulheres } \\
\hline & $\mathrm{D}$ & ND & $\mathrm{D}$ & ND \\
\hline & \multicolumn{4}{|c|}{ Valores médios (kg) para indivíduos de 20 anos } \\
\hline Austrália (Agnew \& Maas, 1982) 28 & 41,0 & 36,0 & 29,0 & 24,1 \\
\hline Estados Unidos (Mathiowetz et al., 1985) ${ }^{14}$ & 54,9 & 47,4 & 31,9 & 27,7 \\
\hline Finlândia (Härkönen et al.,1993) ${ }^{15}$ & 47,5 & - & 30,1 & - \\
\hline Nova Zelândia (Butler, 1997)29 & 58,8 & 59,9 & 35,6 & 32,7 \\
\hline Brasil (Caporrino et al., 1998) $)^{17}$ & 42,8 & 40,7 & 30,0 & 27,2 \\
\hline Estados Unidos (Hanten et al., 1999) ${ }^{18}$ & 54,4 & 49,9 & 31,3 & 28,6 \\
\hline Espanha (Luna-Heredia et al., 2005) ${ }^{23}$ & - & - & - & - \\
\hline \multirow[t]{2}{*}{ Brasil (Schlüssel et al., 2008) ${ }^{30}$} & 45,8 & 43,8 & 27,2 & 25,6 \\
\hline & \multicolumn{4}{|c|}{ Valores médios $(\mathrm{kg})$ para indivíduos de 45 anos } \\
\hline Austrália (Agnew \& Maas, 1982) 28 & 44,0 & 38,0 & 28,5 & 21,8 \\
\hline Estados Unidos (Mathiowetz et al., 1985) ${ }^{14}$ & 49,8 & 45,7 & 28,2 & 25,4 \\
\hline Finlândia (Härkönen et al., 1993) ${ }^{15}$ & 50,8 & - & 30,2 & - \\
\hline Nova Zelândia (Butler, 1997)29 & 51,6 & 49,0 & 35,1 & 33,2 \\
\hline Brasil (Caporrino et al., 1998) ${ }^{17}$ & 44,2 & 39,6 & 32,4 & 29,1 \\
\hline Estados Unidos (Hanten et al., 1999) ${ }^{18}$ & 54,9 & 48,5 & 33,1 & 29,9 \\
\hline Espanha (Luna-Heredia et al., 2005) & 53,0 & 44,5 & 30,2 & 27,9 \\
\hline Brasil (Schlüssel et al., 2008) 30 & 43,2 & 41,6 & 27,0 & 25,7 \\
\hline
\end{tabular}

Nota: D: Mão dominante; ND: Mão não-dominante.

de atividade física dos indivíduos); (v) representatividade da amostra.

É importante frisar, também, que apesar de as amostras desses estudos serem relativamente grandes, todos foram feitos com amostras de conveniência e podem, portanto, não representar as populações de seus países.

\section{Utilização da DM na área da nutrição clínica}

Diversos métodos e ferramentas para avaliação do estado nutricional clínico encontram-se disponíveis hoje. Porém, as baixas sensibilidade e especificidade de alguns desses instrumentos e procedimentos limitam sua utilidade como método de avaliação para determinados pacientes. Há décadas, a avaliação nutricional baseada na antropometria (massa corporal adequada, circunferência muscular do braço, dobras cutâneas) tem sido o método mais amplamente utilizado.

Embora desde o início dos anos 80 venha sendo demonstrada maior sensibilidade de testes funcionais do músculo esquelético à privação nutricional, do que a observada para parâmetros de determinação da composição corpora| ${ }^{31-33}$, sua aplicação na rotina clínica, com o objetivo de auxiliar o acompanhamento nutricional, ainda é discreta. Recentemente, alguns estudos têm comparado parâmetros antropométricos e funcionais, reforçando a validade desses indicadores como um instrumento de avaliação nutricional23,34.

Indicadores funcionais são de particular importância, uma vez que (i) estão correlacionados com complicações clínicas, (ii) a perda de função é um indicador de desnutrição, particularmente a perda de massa corporal magra, e (iii) a recuperação funcional ocorre em poucos dias em resposta ao início de suporte nutricional, em contraste com a recuperação da massa corporal magra, que pode não ocorrer durante a doença ou demorar semanas para se fazer notável durante o período de internação ${ }^{26,35}$. Testes funcionais podem, dessa forma, ser os mais sensíveis e relevantes indicadores de alterações no estado nutricional em curto prazo, bem como da resposta ao suporte nutricional.

Nesse sentido, a DM é descrita como um dos mais sensíveis testes funcionais indicadores 
de depleção protéica ${ }^{1,34}$ e tem sido utilizada como um indicador funcional de desnutrição para esses indivíduos. Para investigar esta hipótese, foi realizada uma busca por referências bibliográficas, utilizando os termos (handgrip or hand-grip or hand grip) and (dynamometry) and (nutrition), na base de dados Medline. Como critérios de inclusão, os artigos deveriam ter, ao menos, o resumo disponível; texto em português, inglês ou espanhol; e a amostra composta por humanos. Não foi aplicado nenhum filtro quanto ao ano de publicação, sexo ou idade dos participantes do estudo. Foram identificados 18 artigos, dentre os quais foram selecionados aqueles referentes à utilização da dinamometria manual como instrumento de avaliação do estado nutricional de pacientes internados. Adicionalmente, buscaram-se algumas das referências citadas nos artigos levantados.

O Quadro 2 apresenta uma síntese de artigos que apontam a DM como um indicador sensível de desnutrição. Alguns estudos apontam baixos valores de DM, apresentados por pacientes internados no período pré-operatório, como um forte indicador de complicações no pós-operatório. A DM é também um bom preditor para detectar o declínio no estado funcional durante o período de internação e, alguns autores, apontam ainda para um maior risco de mortalidade entre pacientes que apresentaram baixos valores desta medida $a^{8,10,35-42}$. Os autores desses estudos concordam em apontar o comprometimento do estado nutricional como responsável pela perda de função do músculo esquelético e, conseqüentemente, pela perda de força manual.

É interessante observar que a metodologia inicialmente descrita por Klidjian et al. ${ }^{1}$ é adotada na maioria desses estudos. Em sua proposta, valores de DM abaixo de $85 \%$ dos valores médios apresentados por uma amostra de indivíduos saudáveis, seriam um indicativo de comprometimento do estado nutricional do paciente internado.

Embora esta metodologia tenha demonstrado ser eficaz na predição de complicações no pós-operatório, para esses indivíduos, não há estudos que descrevam as bases fisiológicas para estabelecer $85 \%$ do valor médio observado por uma amostra de indivíduos saudáveis como ponto de corte entre eutrofia e desnutrição. Além disso, os dados utilizados para comparação dos valores de DM, nesses estudos, são provenientes de amostras pequenas e, em sua maioria, de conveniência; não garantindo, portanto, adequada representatividade para as referidas populações estudadas. Por outro lado, não há base de dados suficiente, que permita estabelecer um outro ponto de corte a partir do qual um indivíduo de determinado sexo e faixa etária estaria classificado como desnutrido segundo seu valor de DM.

Muito da popularidade dos parâmetros antropométricos se deve à facilidade de obtenção e interpretação das medidas; e ao baixo custo para realização das aferições necessárias. A DM surge como uma alternativa tão ou mais simples, objetiva, de baixo custo e pouco invasiva. Contudo, o maior obstáculo à ampla adoção da DM como um instrumento de avaliação nutricional, consiste no fato de que não há uma definição a respeito de um ponto de corte a partir do qual um indivíduo poderia ser classificado como desnutrido.

Segundo Anjos ${ }^{44}$, o uso de dados de uma distribuição de uma medida para determinada população não expressa, necessariamente, o estado de saúde dessa população. Porém, é razoável supor que valores de DM que classifiquem indivíduos, sem outros fatores influenciando seu desempenho muscular, nos percentis mais baixos de uma distribuição populacional para esta variável, possam ser indicativos de alguma redução funcional. Assim, o acompanhamento dessas medidas, para o paciente internado, pode auxiliar na detecção de alterações que não seriam captadas por outros parâmetros no curto prazo.

Para isso, é importante que seja desenvolvido um protocolo de teste padronizado, para que se obtenham medidas válidas. Como explicitado ao longo do texto, diversos fatores não nutricionais podem influenciar esta medida funcional entre os quais, a posição do indivíduo durante a aferição, sua mão de dominância, a motivação em exercer o seu máximo desempenho durante a medida de força, além do instrumento utilizado. Recomenda- 
Quadro 2. Sumário descritivo dos estudos que apontam a dinamometria manual como um instrumento útil de avaliação do estado nutricional para pacientes hospitalizados.

\begin{tabular}{|c|c|c|c|c|c|}
\hline Estudo & $\begin{array}{l}\text { Amostra } \\
\text { avaliada }\end{array}$ & $\begin{array}{c}\text { Dinamômetro } \\
\text { utilizado }^{1}\end{array}$ & Principais resultados & Referência² & $\begin{array}{l}\text { Medida } \\
\text { utilizada }\end{array}$ \\
\hline $\begin{array}{l}\text { Klidjian } \\
\text { et al. }{ }^{1}\end{array}$ & $\begin{array}{l}102 \text { pacientes } \\
\text { (cirurgia } \\
\text { abdominal) }\end{array}$ & $\mathrm{Nl}$ & $\begin{array}{l}\text { Valores de DM abaixo de } 85 \% \text { dos valores médios, observa- } \\
\text { dos em indivíduos saudáveis, foram considerados como indi- } \\
\text { cador de alteração no estado nutricional capaz de predizer } \\
\text { complicações no pós-operatório em } 87 \% \text { dos pacientes que } \\
\text { as desenvolveram. }\end{array}$ & $\begin{array}{l}284 \text { indivíduos } \\
\text { saudáveis }\end{array}$ & $\begin{array}{l}\text { Maior valor } \\
\text { entre } 3 \\
\text { medidas }\end{array}$ \\
\hline $\begin{array}{l}\text { Griffith \& } \\
\text { Clark }^{36}\end{array}$ & $\begin{array}{l}70 \text { pacientes } \\
\text { (cirurgia de } \\
\text { grande porte) }\end{array}$ & $\mathrm{Nl}$ & $\begin{array}{l}\text { Valores de DM inferiores a } 85 \% \text { dos valores esperados, } \\
\text { ajustados para sexo e idade, apresentaram menor valor } \\
\text { preditivo ( } 60 \% \text { vs } 65 \% \text { ), entretanto, maior sensibilidade } \\
\text { ( } 54 \% \text { vs } 39 \% \text { ) e especificidade ( } 85 \% \text { vs } 60 \% \text { ) do que o INP } \\
\text { para complicações no período pós-operatório. }\end{array}$ & $\mathrm{NI}$ & $\begin{array}{l}\text { Maior valor } \\
\text { entre } 3 \\
\text { medidas }\end{array}$ \\
\hline Webb et al. ${ }^{37}$ & $\begin{array}{l}90 \text { pacientes } \\
\text { cirúrgicos }\end{array}$ & Mecânico & $\begin{array}{l}\text { Valores de DM inferiores a } 85 \% \text { dos valores observados em } \\
\text { indivíduos saudáveis, do mesmo sexo e idade, apresenta- } \\
\text { ram alto valor preditivo, em termos de sensibilidade ( } 74 \% \text { ) e } \\
\text { especificidade ( } 51 \%) \text {, para complicações em pacientes ci- } \\
\text { rúrgicos no período pós-operatório. }\end{array}$ & $\begin{array}{l}247 \text { indivíduos } \\
\text { saudáveis }\end{array}$ & $\begin{array}{l}\text { Maior valor } \\
\text { entre } 3 \\
\text { medidas }\end{array}$ \\
\hline $\begin{array}{l}\text { Kalfarentzos } \\
\text { et al. }{ }^{38}\end{array}$ & $\begin{array}{l}95 \text { pacientes } \\
\text { cirúrgicos } \\
\text { (câncer } \\
\text { gastrintestinal) }\end{array}$ & Single Spring & $\begin{array}{l}\text { Valores de DM inferiores a } 85 \% \text { dos valores observados por } \\
\text { indivíduos saudáveis apresentaram maior valor preditivo ( } 58 \% \\
\text { vs } 32 \% \text { ), sensibilidade ( } 78 \% \text { vs } 67 \% \text { ) e especificidade ( } 86 \% \\
\text { vs } 65 \% \text { ) do que o INP para complicações no período } \\
\text { pós-operatório. A DM apresentou uma sensibilidade de } 100 \% \\
\text { para mortalidade nestes pacientes. }\end{array}$ & $\begin{array}{l}240 \text { indivíduos } \\
\text { saudáveis }\end{array}$ & $\begin{array}{l}\text { Maior valor } \\
\text { entre } 3 \\
\text { medidas }\end{array}$ \\
\hline $\begin{array}{l}\text { Hirsch } \\
\text { et al. }{ }^{39}\end{array}$ & $\begin{array}{l}207 \text { pacientes } \\
\text { internados } \\
\text { (127 } \\
\text { cirúrgicos) }\end{array}$ & $\begin{array}{l}\text { Therapeutic } \\
\text { Instruments }\end{array}$ & $\begin{array}{l}\text { Entre os indivíduos classificados como desnutridos segundo } \\
\text { a ASG, a antropometria e a albumina sérica; a DM foi signi- } \\
\text { ficantemente menor do que } 85 \% \text { dos valores apresentados } \\
\text { por indivíduos saudáveis }(p<0,01) \text {. Nenhum dos parâmetros } \\
\text { avaliados foi útil em predizer complicações no período } \\
\text { pós-operatório. }\end{array}$ & $\begin{array}{l}400 \text { indivíduos } \\
\text { saudáveis }\end{array}$ & $\begin{array}{l}\text { Maior valor } \\
\text { entre } 3 \\
\text { medidas }\end{array}$ \\
\hline Guo et al. ${ }^{40}$ & $\begin{array}{l}127 \text { pacientes } \\
\text { internados e } \\
96 \text { cirúrgicos } \\
\text { (câncer oral e } \\
\text { maxilofacial) }\end{array}$ & $\mathrm{Nl}$ & $\begin{array}{l}\text { Houve forte correlação positiva entre a DM e CMB (homens: } \\
r=0,596 ; p<0,01 \text { e mulheres: } r=0,565 ; p<0,01) \text { e o ICE (ho- } \\
\text { mens: } r=0,661 ; p<0,01 \text { e mulheres: } r=0,601 ; p<0,01 \text { ). Paci- } \\
\text { entes que apresentaram valores de DM inferiores a } 85 \% \\
\text { dos valores observados em indivíduos saudáveis, desenvol- } \\
\text { veram significantemente mais complicações do que aqueles } \\
\text { que apresentaram } 85 \% \text { ou mais }(15 / 31 \text { [48\%] vs } 12 / 65 \\
[18 \%], p<0,004) \text {. }\end{array}$ & $\begin{array}{l}203 \text { indivíduos } \\
\text { saudáveis }\end{array}$ & $\begin{array}{l}\text { Maior valor } \\
\text { entre } 3 \\
\text { medidas }\end{array}$ \\
\hline $\begin{array}{l}\text { Qureshi } \\
\text { et al. }{ }^{8}\end{array}$ & $\begin{array}{l}128 \text { pacientes } \\
\text { em } \\
\text { hemodiálise }\end{array}$ & Harpenden & $\begin{array}{l}\text { Nos grupos de pacientes classificados como levemente e } \\
\text { moderadamente/severamente desnutridos segundo ASG, os } \\
\text { valores de DM foram significantemente menores do que os } \\
\text { apresentados pelo grupo controle }(p<0,01) \text {. Ao comparar os } \\
\text { grupos de pacientes em hemodiálise, observou-se uma ten- } \\
\text { dência linear de diminuição dos valores de DM conforme } \\
\text { aumentava o grau de desnutrição }(p<0,001) \text {. }\end{array}$ & $\begin{array}{l}44 \text { indivíduos } \\
\text { saudáveis }\end{array}$ & $\begin{array}{l}\text { Maior valor } \\
\text { entre } 3 \\
\text { medidas }\end{array}$ \\
\hline $\begin{array}{l}\text { Lê Cornu } \\
\text { et al. }{ }^{9}\end{array}$ & $\begin{array}{l}82 \text { pacientes } \\
\text { cirúrgicos } \\
\text { (transplante } \\
\text { de fígado) }\end{array}$ & Harpenden & $\begin{array}{l}\text { Valores de DM abaixo de } 85 \% \text { dos valores apresentados por } \\
\text { indivíduos saudáveis, ajustados para sexo e idade, foram o } \\
\text { único marcador do estado nutricional que esteve associado } \\
\text { com a ocorrência de complicações no pós-operatório }(p=0,05) \text {. }\end{array}$ & $\mathrm{NI}$ & $\begin{array}{l}\text { Maior valor } \\
\text { entre } 3 \\
\text { medidas }\end{array}$ \\
\hline $\begin{array}{l}\text { Figueiredo } \\
\text { et al. }{ }^{34}\end{array}$ & $\begin{array}{l}69 \text { pacientes } \\
\text { internados } \\
\text { (hepatopatas } \\
\text { terminais) }\end{array}$ & Jamar & $\begin{array}{l}\text { A DM apresentou correlação positiva moderada com o IMC } \\
(r=0,54) \text {. Pacientes com valores de IMC no último quartil de } \\
\text { distribuição para o sexo apresentaram menores valores de } \\
\text { DM do que os demais }(p<0,001) \text {. Na análise multivariada, } \\
\text { observou-se que a CMB e a DM foram os melhores fatores } \\
\text { preditivos de IMC. Valores de DM }<30 \mathrm{~kg} \text { e CMB }<23 \mathrm{~cm} \\
\text { combinados apresentaram sensibilidade de } 94 \% \text { e valor } \\
\text { preditivo positivo de } 97 \% \text { em identificar pacientes com } \\
\text { depleção do estado nutricional segundo o IMC. }\end{array}$ & $\begin{array}{l}\text { Valores preditos } \\
\text { pelas equações } \\
\text { propostas pelo } \\
\text { NSDC }\end{array}$ & $\begin{array}{l}\text { Média de } \\
3 \text { medidas }\end{array}$ \\
\hline
\end{tabular}


Quadro 2. Sumário descritivo dos estudos que apontam a dinamometria manual como um instrumento útil de avaliação do estado nutricional para pacientes hospitalizados.

\begin{tabular}{|c|c|c|c|c|c|}
\hline Estudo & $\begin{array}{l}\text { Amostra } \\
\text { avaliada }\end{array}$ & $\begin{array}{l}\text { Dinamômetro } \\
\text { utilizado }^{1}\end{array}$ & Principais resultados & Referência ${ }^{2}$ & $\begin{array}{l}\text { Medida } \\
\text { utilizada }\end{array}$ \\
\hline $\begin{array}{l}\text { Figueiredo } \\
\text { et al. }{ }^{41}\end{array}$ & $\begin{array}{l}53 \text { pacientes } \\
\text { cirúrgicos } \\
\text { (transplante de } \\
\text { fígado) }\end{array}$ & Jamar & $\begin{array}{l}\text { Menores valores de DM no período pré-operatório esti- } \\
\text { veram associados com maior tempo de permanência na } \\
\text { UTI }(p<0,01) \text {, mas não com episódios de infecção pós- } \\
\text { operatórios; rejeição do órgão transplantado e morte. }\end{array}$ & NA & $\begin{array}{l}\text { Média de } 3 \\
\text { medidas }\end{array}$ \\
\hline $\begin{array}{l}\text { Humphreys } \\
\text { et al. }{ }^{42}\end{array}$ & $\begin{array}{l}50 \text { pacientes } \\
\text { internados ( } 25 \\
\text { cirúrgicos) }\end{array}$ & $\begin{array}{l}\text { Therapeutic } \\
\text { Instruments }\end{array}$ & $\begin{array}{l}\text { Observou-se correlação positiva fraca }(r=0,36 ; p=0,019) \text {, } \\
\text { entre a DM e alterações no valor da massa corporal } \\
\text { durante a internação. Menores valores de DM foram } \\
\text { observados entre pacientes classificados como "risco de } \\
\text { desnutrição" e "severamente desnutridos", segundo a } \\
\text { ASG; entretanto essas diferenças não foram estatistica- } \\
\text { mente significantes. A DM foi o melhor fator preditivo } \\
\left.\text { da evolução clínica ( } \beta=0,448 ; r^{2}=0,568 ; p<0,001\right) \text {, defini- } \\
\text { da como um escore do estado funcional durante a } \\
\text { internação. }\end{array}$ & $\begin{array}{l}400 \text { indivíduos } \\
\text { saudáveis }\end{array}$ & $\begin{array}{l}\text { Maior valor } \\
\text { entre } 3 \\
\text { medidas }\end{array}$ \\
\hline $\begin{array}{l}\text { Álvares-da- } \\
\text { Silva \& da } \\
\text { Silveira }^{43}\end{array}$ & $\begin{array}{l}50 \text { pacientes } \\
\text { hepatopatas }\end{array}$ & Kratos & $\begin{array}{l}\text { Usando a ASG como "padrão ouro", a DM apresentou } \\
\text { sensibilidade de } 100 \% \text { e especificidade de } 46 \% \text { na } \\
\text { detecção de desnutrição. A DM foi o único indicador } \\
\text { do estado nutricional capaz de predizer a ocorrência de } \\
\text { complicações em um ano de seguimento }(p<0,05) \text {. }\end{array}$ & $\begin{array}{l}108 \text { indivíduos } \\
\text { saudáveis }\end{array}$ & $\begin{array}{l}\text { Maior valor } \\
\text { entre } 3 \\
\text { medidas }\end{array}$ \\
\hline $\begin{array}{l}\text { Wang } \\
\text { et al. }{ }^{11}\end{array}$ & $\begin{array}{l}233 \text { pacientes } \\
\text { nefropatas }\end{array}$ & Smedley & $\begin{array}{l}\text { Tanto homens quanto mulheres que morreram ao lon- } \\
\text { go do seguimento apresentaram menores valores de } \\
\text { DM do que aqueles que permaneceram vivos }(p<0,001) \text {. } \\
\text { Após ajuste para fatores de confusão, a DM foi um for- } \\
\text { te fator preditivo de morte por todas as causas (RC: } \\
0,95 \text {; IC: } 0,92-0,99 ; p=0,005) \text {. }\end{array}$ & NA & $\begin{array}{l}\text { Maior valor } \\
\text { entre } 3 \\
\text { medidas }\end{array}$ \\
\hline
\end{tabular}

1 A informação sobre o instrumento de aferição da DM não foi fornecida de forma padronizada pelos estudos revisados. Essas informações estão disponíveis na forma de tipo e modelo do dinamômetro, quando possível; ${ }^{2}$ Indivíduos avaliados especificamente para fornecer dados de comparação. Siglas: ASG: avaliação subjetiva global; CMB: circunferência muscular do braço; DM: dinamometria manual; IC: intervalo de confiança de 95\%; ICE: índice creatinina-estatura; IMC: índice de massa corporal; INP: índice nutricional prognóstico; NA: não se aplica; NI: não informado; NSDC: the National Strength Database Consortium; RC: razão de chances; UTI: unidade de Tratamento Intensivo.

se que as medidas de DM sejam feitas com os indivíduos de pé, quando possível, com os braços não flexionados e paralelos ao corpo, observando o maior valor entre três medidas repetidas e com um intervalo de, no mínimo, um minuto para cada medida.

Compreender o comportamento da variável DM na população seria um primeiro passo importante para estabelecer um ponto de corte a partir do qual indivíduos poderiam ser classificados como desnutridos com base nos valores apresentados para esta variável. Estabelecer curvas com valores de distribuição percentilar para DM, obtidas de uma amostra de base populacional pode contribuir para esse entendimento ${ }^{30}$. Além disso, tais curvas poderiam servir como base para comparações entre as medidas de DM observadas em populações de diferentes países ou grupos étnicos.

\section{COLABORADORES}

M.M. SCHLÜSSEL participou de todas as etapas do estudo, desde o planejamento da revisão, levantamento bibliográfico, seleção dos artigos, análise dos resultados até a elaboração final do artigo. L.A. ANJOS 
participou do planejamento da revisão e contribuiu no levantamento bibliográfico e nas revisões de todas as versões do artigo. G. KAC contribuiu na orientação e revisão de todas as versões do artigo e na revisão da versão final.

\section{REFERÊ NCIAS}

1. Klidjian AM, Foster KJ, Kammerling RM, Cooper A, Karran SJ. Relation of anthropometric and dynamometric variables to serious postoperative complications. BMJ. 1980; 281(6245):899-901.

2. Bohannon RW. Hand-grip dynamometry provides a valid indication of upper extremity strength impairment in home care patients. J Hand Ther. 1998; 11(4): 258-60.

3. Bohannon RW. Adoption of hand-held dynamometry. Percept Mot Skills. 2001; 92(1):150.

4. Bohannon RW. Dynamometer measurements of hand-grip strength predict multiple outcomes. Percept Mot Skills. 2001; 93(2):323-8.

5. Stalenhoef PA, Diederiks JPM, Knottnerus JA, Kester ADM, Crebolder HFJM. A risk model for the prediction of recurrent falls in community-dwelling elderly: a prospective cohort study. J Clin Epidemiol. 2002; 55(11):1088-94.

6. Innes E. Handgrip strength testing: a review of literature. Aust Occup Ther J. 1999; 46(3):120-40.

7. Vaz M, Thangam S, Prabhu A, Shetty PS. Maximal voluntary contraction as a functional indicator of adult chronic undernutrition. Br J Nutr. 1996; 76(1):9-15.

8. Qureshi AR, Alvestrand A, Danielsson A, DivinoFilho JC, Gutierrez A, Lindholm B, et al. Factors predicting malnutrition in hemodialysis patients: a cross-sectional study. Kidney Int. 1998; 53(3): 773-82.

9. Le Cornu KA, McKiernan FJ, Kapadia SA, Neuberger JM2nd. A prospective randomized study of preoperative nutritional supplementation in patients awaiting elective orthotopic liver transplantation. Transplantation. 2000; 69(7): 1364-9.

10. Teraoka T. Studies on the peculiarity of grip strength in relation to body positions and aging. Kobe J Med Sci. 1979; 25(1):1-17.

11. Wang AY, Sea MM, Ho ZS, Lui S, Li PK, Woo J. Evaluation of handgrip strength as a nutritional marker and prognostic indicator in peritoneal dialysis patients. Am J Clin Nut. 2005; 81(1):79-86.

12. Watanabe W, Owashi K, Kanauchi Y, Mura N, Takahara M, Ogino T. The short-term reliability of grip strength measurement and the effects of posture and grip span. J Hand Surg [Am]. 2005; 30(3):603-9.

13. Schmidt RT, Toews JV. Grip strength as measured by the Jamar dynamometer. Arch Phys Med Rehab. 1970; 51(6):321-7.

14. Mathiowetz V, Kashman N, Volland G, Weber K, Dowe M, Rogers S. Grip and pinch strength: normative data for adults. Arch Phys Med Rehab. 1985; 66(2):69-72.

15. Härkönen R, Piirtomaa M, Alaranta H. Grip strength and hand position of the dynamometer in 204 Finnish adults. J Hand Surg [Br]. 1993; 18(1):129-32.

16. Crosby CA, Wehbé MA, Mawr B. Hand strength: normative values. J Hand Surg. 1994; 19(4):665-70.

17. Caporrino FA, Faloppa F, Santos JBG, Réssio C, Soares FHC, Nakachima LR, et al. Estudo populacional de força de preensão palmar com dinamômetro Jamar ${ }^{\circledR}$. Rev Bras Ortop. 1998; 33(2): 150-4.

18. Hanten WP, Chen WY, Austin AA, Brooks RE, Carter $H C$, Law CA, et al. Maximum grip strength in normal subjects from 24 to 64 years of age. J Hand Ther. 1999; 12:193-200.

19. Incel NA, Ceceli E, Durukan PB, Erdem HR, Yorgancioglu ZR. Grip strength: Effect of hand dominance. Singapore Med J. 2002; 43(5):234-7.

20. Boadella JM, Kuijer PP, Sluiter JK, Frings-Dresen $\mathrm{MH}$. Effect of self-selected handgrip position on maximal handgrip strength. Arch Phys Med Rehab. 2005; 86(2):328-31.

21. Bechtol CO. The use of a dynamometer with adjustable handle spacings. J Bone Joint Surg Am. 1954; 36(4):820-4; 32.

22. Armstrong CA, Oldham JA. A comparison of dominant and nondominant hand strengths. J Hand Surg [Br]. 1999; 24(4):421-5.

23. Luna-Heredia E, Martín-Peña G, Ruiz-Galiana J. Handgrip dynamometry in healthy adults. Clin Nutr. 2005; 24(2):250-8.

24. Hillman TE, Nunes QM, Hornby ST, Stanga Z, Neal $\mathrm{KR}$, Rowlands BJ, et al. A practical posture for hand grip dynamometry in the clinical setting. Clin Nutr. 2005; 24(2):224-8.

25. Johansson CA, Kent BE, Shepard KF. Relationship between verbal command volume and magnitude of muscle contraction. Phys Ther. 1983; 63(8): 1260-5.

26. Hornby ST, Nunes QM, Hillman TE, Stanga Z, Neal $K R$, Rowlands BJ, et al. Relationships between structural and functional measures of nutritional status in a normally nourished population. Clin Nutr. 2005; 24(3):421-6. 
27. Trossman PB, Li PW. The effect of the duration of intertribal rest periods on isometric grip strength performance in young adults. Occup Ther J Res. 1989; 9:362-78.

28. Agnew P, Maas F. An interim Australian version of the Jebsen test of hand function. Aust J Physiother. 1982; 28(2):23-9.

29. Butler M. Grip strength: a comparative study. New Zealand J Occup Ther. 1997; 48:5-12.

30. Schlüssel MM, Anjos LA, Vasconcellos MTL, Kac G. Referente values of handgrip dynamometry of health adults: A populatin-based study. Clin Nutr. 2008; doi: 10.1016/j.clin.2008.04.004.

31. Lopes J, Russell DM, Whitwell J, Jeejeebhoy, KN. Skeletal muscle function in malnutrition. Am J Clin Nutr. 1982; 36(4):602-10.

32. Russell DM, Leiter LA, Whitwell J, Marliss EB, Jeejeebhoy KN. Skeletal muscle function during hypocaloric diets and fasting: a comparison with standard nutritional assessment parameters. Am J Clin Nutr. 1983; 37(1):133-8.

33. Jeejeebhoy KN. Muscle function and nutrition. Gut. 1986; 27(S1):25-39.

34. Figueiredo FA, Dickson ER, Pasha TM, Porayko MK, Therneau TM, Malinchoc M, et al. Utility of standard nutritional parameters in detecting body cell mass depletion in patients with end-stage liver disease. Liver Transpl. 2000; 6(5):575-81.

35. Russell DM, Prendergast PJ, Darby PL, Garfinkel PE, Whitwell J, Jeejeebhoy KN. A comparison between muscle function and body composition in anorexia nervosa: the effect of refeeding. Am J Clin Nutr. 1983; 38(2):229-37.

36. Griffith CDM, Clark RG. A comparison of the 'Sheffield' prognostic index with forearm muscle dynamometry in patients from Sheffield undergoing major abdominal and urological surgery. Clin Nutr. 1984; 3(3):147-51.
37. Webb AR, Newman LA, Taylor M, Keogh JB. Hand grip dynamometry as a predictor of postoperative complications reappraisal using age standardized grip strengths. JPEN J Parenter Enteral Nutr. 1989; 13(1):30-3.

38. Kalfarentzos F, Spiliotis J, Velimezis G, Dougenis D, Androulakis J. Comparison of forearm muscle dynamometry with nutritional prognostic index, as a preoperative indicator in cancer patients. JPEN J Parenter Enteral Nutr. 1989; 13(1):34-6.

39. Hirsch S, De la Maza MP, Obaldia N, Espinoza J, Hübner $C$, Petermann $M$, et al. Fuerza muscular: um indicador de estado nutritivo. Rev Med Chile. 1992; 120(6):615-20.

40. Guo C, Zhang W, Ma D, Zhang K, Huang J. Hand grip strength: an indicator of nutritional state and the mix of postoperative complications in patients with oral and maxillofacial cancers. Br J Oral Maxillofac Surg. 1996; 34(4):325-7.

41. Figueiredo F, Dickson ER, Pasha T, Kasparova $P$, Therneau T, Malinchoc $M$, et al. Impact of nutritional status on outcomes after liver transplantation. Transplantation. 2000; 70(9): 1347-52.

42. Humphreys J, De la Maza P, Hirsch S, Barrera G, Gattas $V$, Bunout D. Muscle strength as a predictor of loss of functional status in hospitalized patients. Nutrition. 2002; 18(8):616-20.

43. Álvares-da-Silva MR, Silveira TR. Comparison between handgrip strength, subjective global assessment, and prognostic index in assessing malnutrition and predicting clinical outcome in cirrhotic outpatients. Nutrition. 2005; 21(2):113-7.

44. Anjos LA. Obesidade e saúde pública. Rio de Janeiro: Fiocruz; 2006. 